\title{
Los Objetivos de Desarrollo Sostenible y las capacidades tecnológicas
}

\author{
David Mayer-Foulkes ${ }^{1}$, Edson Serván-Mori y Gustavo Nigenda ${ }^{3}$
}

Forma de citar

Mayer-Foulker D, Serván-Mori E, Nigenda G. Los Objetivos de Desarrollo Sostenible y las capacidades tecnológicas. Rev Panam Salud Publica. 2020;44:e141. https://doi.org/10.26633/RPSP.2020.141

RESUMEN

Para lograr los objetivos de desarrollo y salud sostenibles, es esencial incrementar las capacidades tecnológicas de las poblaciones más desfavorecidas. Entrado el siglo XXI, existen las tecnologías necesarias para ello. El déficit en capacidades tecnológicas se debe a la existencia de un gradiente tecnológico entre la producción de gran y de pequeña escalas, debido a la falta de incentivos para la innovación y la difusión en empresas y comunidades pequeñas. En estos ámbitos el cambio tecnológico, punto medular del desarrollo, es un bien público que la economía de mercado no provee eficientemente. Su provisión requiere la aplicación de políticas públicas de innovación y difusión tecnológicas. La reducción del gradiente tecnológico constituye, pues, parte medular de la Agenda 2030 para el Desarrollo Sostenible, de las Naciones Unidas, y la Agenda de Salud Sostenible para las Américas 2018-2030, de la Organización Panamericana de la Salud. Esto es aplicable, así mismo, al desarrollo de los sistemas de salud, que funcionan también como mecanismos de redistribución para romper las trampas de pobreza. Asimismo, las experiencias en esos sistemas tienen relevancia para aplicar políticas de incremento de capacidades tecnológicas que disminuyan la pobreza, mejoren los determinantes sociales de la salud y, con ello, reduzcan la magnitud de la trampa de desarrollo humano.

Palabras clave Ciencia, tecnología y sociedad; desarrollo sostenible; equidad; sistemas de salud; política de salud; determinantes sociales de la salud.

Los Objetivos de Desarrollo Sostenible (ODS) establecidos en la Agenda 2030 para el Desarrollo, de las Naciones Unidas (1), y la Agenda de Salud Sostenible para las Américas 2018-2030, promulgada por la Organización Panamericana de la Salud (2), plantean reducir la pobreza, promover la industrialización y el desarrollo sostenible, y alcanzar el máximo nivel de salud y equidad posibles. Estos objetivos multisectoriales son integrados e indivisibles, pues dependen mutuamente entre sí, como se señala en el marco de los determinantes sociales de la salud (DSS) $(3,4)$.

El desarrollo económico y humano, y su distribución, incluidos los salarios reales, reflejan directamente las diferencias

\footnotetext{
Centro de Investigación y Docencia Económicas, México. $\square$ david.mayer@ cide.edu

2 Centro de Investigación en Sistemas de Salud. Instituto Nacional de Salud Pública, México.

3 Escuela Nacional de Enfermería y Obstetricia de México. Universidad Nacional Autónoma de México, México.
}

en el nivel tecnológico ${ }^{a}$, tanto entre países como al interior de ellos. Lograr el desarrollo tecnológico con equidad es una condición necesaria tanto para sostener el acceso al trabajo suficientemente productivo y remunerado, como para lograr los ODS. En este artículo se muestra cómo el desarrollo tecnológico, particularmente en la producción de pequeña escala, vinculada con la población de bajos ingresos, tiene una naturaleza de bien público y, por lo tanto, su provisión a través del mercado es ineficiente; de ahí su estrecho vínculo con los ODS.

Siguiendo el concepto de capacidades de Sen y Nussbaum, estas deben trascender del nivel individual al social. Por

\footnotetext{
a Se entiende por tecnología los procedimientos utilizados para convertir insumos en productos (no solo en la informática). El desarrollo tecnológico es el avance en estas técnicas de producción.
} 
ejemplo, ejercer el derecho al trabajo por sí mismo no es bastante, ya que este debe ser suficientemente productivo, y esto es un atributo social, no solamente individual o privado. Es decir, las capacidades tecnológicas son un atributo social. De hecho, lo mismo sucede con la educación y la salud, cuyos mercados y distribución también operan con fallas.

En las sociedades industriales modernas, el cambio tecnológico en empresas grandes con producción a escala reditúa más que en empresas pequeñas y avanza con más empuje la tecnología de las empresas grandes que la de las pequeñas. Como resultado, se genera un gradiente tecnológico - es decir, diferencias de productividad- entre los sectores de gran y de pequeña escalas $(5,6)$. Surge un atraso relativo en la productividad de las empresas de menor escala, que emplean a una parte importante de la población. Esta distribución desigual de la productividad se relaciona directamente con la distribución desigual del ingreso. La asociación entre desigualdad de ingreso y de esperanza de vida ha sido ya demostrada (7).

Las siguientes cifras ilustran el gradiente tecnológico y salarial característico de las economías industriales. En México, en 2013, la remuneración anual promedio en las microempresas con diez empleados o menos - que ocupaban al 20,8\% de los trabajadores- fue de MXN 53 500, mientras que en las empresas con más de 251 empleados — que ocupaban al 53,6\% de los trabajadores- fue de MXN 169 500, 3,17 veces mayor (8). En los Estados Unidos de América, en 2017, las empresas manufactureras con producción menor de US\$100 millones - con 19,1 empleados en promedio y que ocupaban al 39,4\% de los trabajadores - pagaban un salario promedio de US $\$ 47397$, mientras que las de producción mayor de US\$2 500 millones con 5657,4 empleados en promedio y que ocupaban al 30,8\% de los trabajadores- pagaban US\$71 666, es decir, 1,51 veces más (9). Como se observa, la desigualdad salarial fue proporcionalmente mayor en México que en los Estados Unidos.

A continuación, se analiza la naturaleza de bien público del desarrollo tecnológico y se explica cómo se genera el gradiente tecnológico. Se señala el papel dinámico básico del gradiente tecnológico en los DSS y se ejemplifica con la trampa de desarrollo humano ${ }^{\mathrm{b}}$, explicando cómo se relaciona su magnitud con el gradiente tecnológico y el déficit en capacidades tecnológicas. Finalmente, se analiza el impacto mutuo entre las políticas de gestión de los sistemas de salud y las de aumento de capacidades tecnológicas.

\section{EL GRADIENTE EN CAPACIDADES TECNOLÓGICAS}

El cambio en cualquier tipo de técnica de producción, es decir, el cambio tecnológico, consiste principalmente en crear conocimiento. Puesto que el conocimiento es un bien público, invertir en crearlo es redituable solamente si su beneficio es apropiable. En empresas grandes es más factible esta apropiación, posiblemente facilitada por patentes, y la magnitud del beneficio justifica una mayor inversión. En cambio, en empresas pequeñas es más probable que se conozcan o difundan las innovaciones, lo que reduce el beneficio apropiable, y puede no justificarse la inversión. Sin embargo, la creación de ese bien público podría justificarse para el sector

En una trampa de pobreza, las familias pertenecen a equilibrios diferentes, más altos o bajos, sin que la dinámica de mercado disuelva las diferencias. productivo en su conjunto. Si bien incluso en sectores de gran escala la inversión en cambio tecnológico puede ser subóptima, en sectores de pequeña escala esta puede permanecer como bien público potencial, sin incentivos para realizarse. En estos sectores no se produce el cambio tecnológico, que queda limitado a la compra de insumos innovadores producidos en empresas grandes o a nuevos conocimientos del personal calificado.

En resumen, es más intensa la innovación en sectores de gran escala que en sectores de pequeña escala, ya que en el ámbito de las pequeñas empresas y comunidades, la economía de mercado no provee suficientes incentivos para la innovación y la difusión tecnológicas. Esta dinámica genera un gradiente tecnológico, tanto en investigación y desarrollo como en difusión tecnológica. Las diferencias tecnológicas surgen debido a diferentes ritmos de innovación en sectores de diferentes escalas. Este argumento se ha formalizado previamente $(5,6)$. La magnitud del gradiente tecnológico varía entre ámbitos y países, y es más pronunciado donde es mayor la desigualdad.

Las empresas grandes pueden financiar sus propios desarrollos tecnológicos mediante la utilización de personal calificado, la investigación científica y otras formas de colaboración social. En el caso de las empresas pequeñas, esta colaboración es incluso más necesaria. La educación y la ciencia pueden crear y difundir la tecnología. Los sectores productivo y educativo-científico deben vincularse con todo el espectro productivo y tecnológico, tanto de pequeña como de gran escala. Desde su formación, los estudiantes de diversas carreras pueden integrar un currículo de actividades significativas en los sectores productivo y de salud, que se incorpore a su formación y futuras posibilidades de empleo. Tanto el contenido de los programas y la docencia como las tecnologías que ocupan productores pequeños, medianos y grandes, pueden mejorarse en este proceso, prácticamente sin costo adicional, mediante la integración de los criterios adecuados al desarrollo de las actividades existentes. También puede incentivarse la innovación que beneficie a los sectores menos favorecidos, ya sea en la producción de los satisfactores de las necesidades básicas —incluida la vivienda, la alimentación, la salud, el agua, el drenaje, la sustentabilidad- como en sus diversos ámbitos de trabajo. La gran diversidad de tecnologías que existe actualmente puede aplicarse a resolver todo tipo de problemas.

Lograr este fortalecimiento de capacidades tecnológicas, que depende de la acción pública, reduce la pobreza e incrementa las posibilidades de una vida saludable. El aumento en el ingreso de los sectores económicos de menor renta, logrado con la mejora de sus fundamentos productivos, provocará un efecto multiplicador que hará crecer todos los sectores, lo que generará un impulso de desarrollo integral con efectos positivos en el logro de los ODS.

\section{GRADIENTE TECNOLÓGICOY DETERMINANTES SOCIALES DE LA SALUD}

Los elementos descritos permiten sugerir que el desarrollo tecnológico constituye el trasfondo dinámico de los determinantes del proceso salud-enfermedad, fundamental en la identificación, el diseño y la implantación de estrategias efectivas de largo plazo en favor del bienestar social. 
Un menor gradiente tecnológico promueve resultados positivos en bienestar y salud, aumenta el acceso a bienes básicos y servicios primarios de atención de la salud, y mitiga las inequidades en salud, lo que genera desarrollo político y cultural, y madurez institucional. El desarrollo tecnológico influye en la salud a través de las estructuras sociales de oportunidad y acceso a recursos, y como mecanismo de redistribución de los avances en la salud.

Diversos campos del conocimiento han analizado el proceso salud-enfermedad y las complejas relaciones que tiene con sus determinantes, desde una visión biológica del proceso, como en las ciencias biomédicas, hasta una visión socio-ecológica, que explicita los factores no biológicos que explican las diferencias relacionadas con la salud (10). Sin embargo, ninguna de ellas toma en cuenta la distribución tecnológica y su dinámica. Las políticas actuales de salud tampoco lo hacen $(11,12)$.

El vínculo dinámico tecnología-salud expande el marco de los DSS (13) al identificar que las condiciones en las que la población nace, vive, trabaja, envejece y muere expresan sus capacidades tecnológicas, las que a su vez dependen de la organización y el posicionamiento sociales $(3,4)$. Los DSS son todas las características sociales que afectan a la salud (13). Los grupos poblacionales en desventaja experimentan una salud más precaria derivada de la desigualdad social y el acceso diferencial a recursos básicos, así como por el perfil de capacidades poblacionales (por ejemplo, la escolaridad, el desempleo, etc.), que definen en conjunto su capacidad tecnológica, en el nivel social señalado previamente. La relevancia de los DSS ha sido ampliamente reconocida $(13,14)$.

En la figura 1 se muestra una adaptación del marco conceptual propuesto por la Comisión de los Determinantes Sociales de Salud de la Organización Mundial de la Salud a partir de la propuesta de Solar e Irwin (3), que incluye el componente tecnológico, tanto en un sentido estático como dinámico. Estáticamente, la tecnología se ubica como parte de los determinantes estructurales (económicos, políticos, culturales y sociales); a su vez, el gradiente tecnológico modela los determinantes intermedios y próximos. Dinámicamente, el cambio tecnológico - y su distribución- es un factor fundamental de los determinantes estructurales e intermedios. Reducir el gradiente tecnológico y con ello incrementar las capacidades tecnológicas es esencial para reducir la inequidad en los DSS. El sistema de salud forma parte de los determinantes intermedios, ya que condiciona la distribución de las oportunidades de acceso a los servicios de salud y la calidad con la que los servicios son provistos, con implicaciones sobre la vulnerabilidad financiera, particularmente en las poblaciones que viven en condiciones de pobreza. En última instancia, el gradiente tecnológico modela la forma y el retraso relativo con los que interactúan las personas, el sistema de salud y la información. En este sentido, la maximización del efecto de los desarrollos tecnológicos sobre la salud poblacional dependerá estrechamente de su implementación sostenible y equitativa, bajo principios de colaboración local $\mathrm{y}$ acorde con sus principios éticos y valores.

\section{DESARROLLO HUMANO Y GRADIENTE TECNOLÓGICO}

El papel dinámico del gradiente tecnológico en los DSS se puede abordar mediante el desarrollo humano. Este requiere de inversiones durante la niñez y la juventud —como en educación y salud - que padres con niveles bajos de desarrollo humano frecuentemente no pueden realizar, a pesar de ser necesarios. En consecuencia, las desigualdades, o gradiente en el desarrollo humano, tienden a transmitirse entre generaciones (15). Esta

FIGURA 1. Tecnología como determinante social de la salud

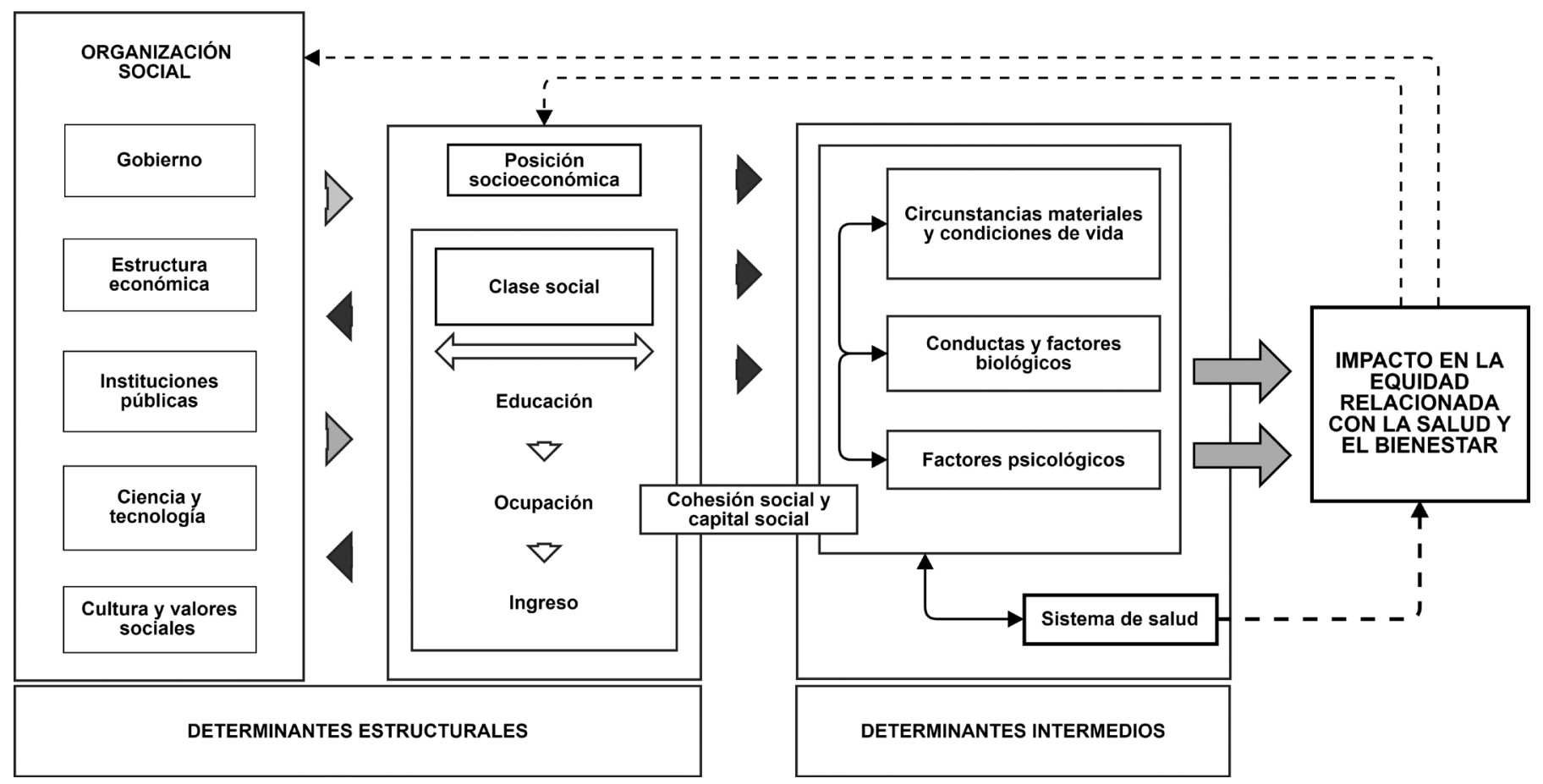

Fuente: Elaboración propia a partir de datos de la referencia 3. 


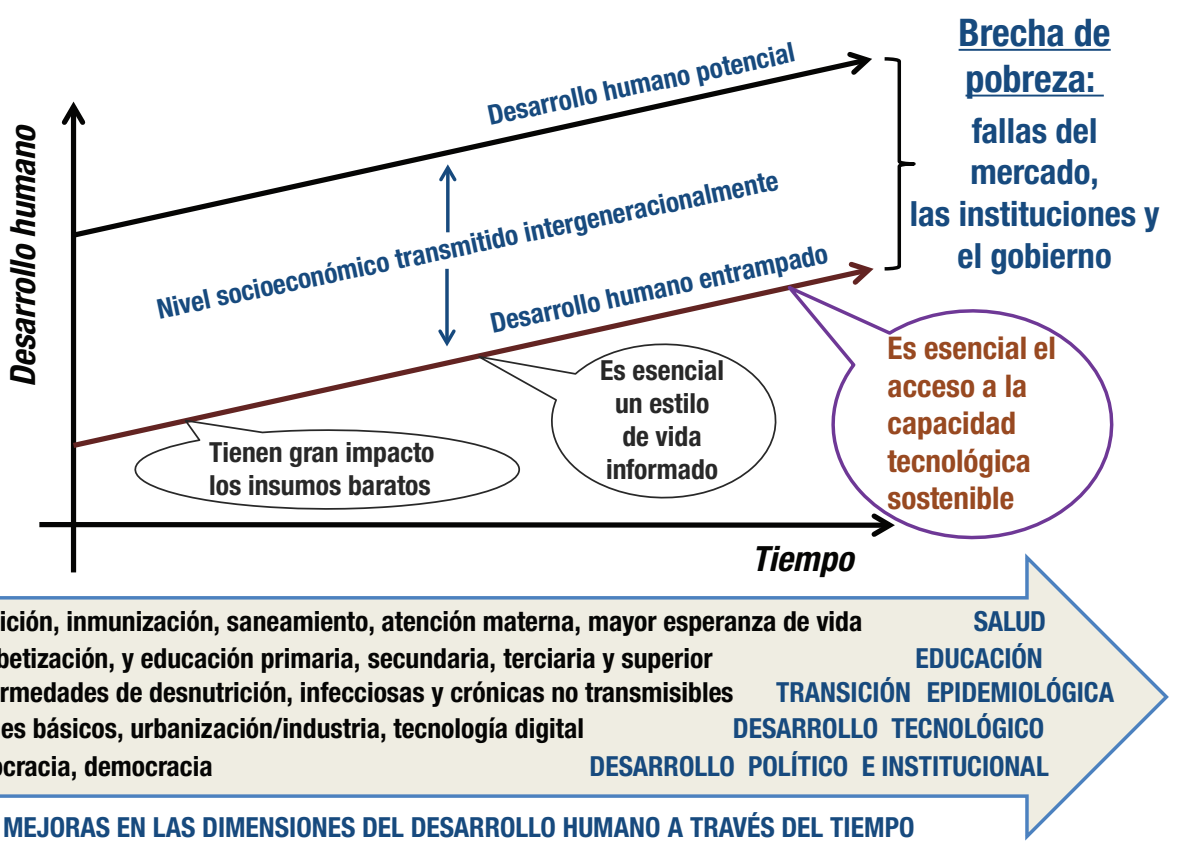

Fuente: Elaboración de los autores a partir de Mayer-Foulkes y Pescetto-Villouta (17), con autorización de Walter de Gruyter GmbH, a través del Copyright Clearance Center, Inc.

La figura muestra la distribución del desarrollo humano a lo largo del tiempo, modelada como resultado de una trampa de pobreza dinámica en un contexto de cambio tecnológico diferenciado por niveles socioeconómicos. La trampa de pobreza se debe a fallas de mercado e institucionales intergeneracionales. En el eje vertical, se muestra una población estratificada: los más pobres viven con un nivel tecnológico más bajo y no logran dotar a sus hijos con capital humano suficiente para lograr los niveles de desarrollo humano potenciales de su sociedad. Aunque los niveles tecnológicos y de desarrollo humano se incrementan con el tiempo, subsisten rezagos significativos. La profundidad vertical de la trampa de desarrollo humano es función del gradiente tecnológico. En el eje horizontal, se muestra la transición epidemiológica de enfermedades contagiosas y nutricionales a enfermedades crónicas no transmisibles. Se transita de tecnologías relativamente simples y directas, como las vacunas y el saneamiento, hacia la provisión y distribución de otras tecnologías, como la alimentación sana y los estilos de vida saludables, que conllevan aspectos tanto colectivos como individuales. Una siguiente etapa requiere del acceso a capacidades tecnológicas sostenibles.

situación constituye una falla del mercado que crea una trampa de pobreza que, a su vez, perpetúa una distribución injusta del desarrollo humano a través de la estructura social. Cuando esta trampa subsiste en un contexto de desarrollo tecnológico se denomina trampa de desarrollo humano $(16,17)$.

En la figura 2 se presenta esa trampa (17), que incluye, entre otros elementos, la transición epidemiológica que implica un cambio de patrón en la mortalidad y la morbilidad, de enfermedades contagiosas y nutricionales a enfermedades crónicas no transmisibles. El siglo XXI presenta una siguiente transición, en la que las capacidades tecnológicas resultan los determinantes principales de la salud, particularmente en los países de ingresos medios y bajos. En todos los rubros básicos — vivienda, alimentación, sanidad, medicina, educación y trabajo-, la tecnología está lo suficientemente avanzada como para que la población en su conjunto goce de altos niveles de salud y bienestar. Así, el incremento en la salud y el bienestar de la población depende cada vez más de su capacidad tecnológica. Resalta cada vez más la importancia de la desigualdad en estas capacidades, incluido el acceso al trabajo productivo, es decir, el gradiente tecnológico.

En resumen, la profundidad de la trampa de desarrollo humano y los rezagos en los ODS son resultados de la magnitud del gradiente tecnológico.

\section{SISTEMAS DE SALUD: TECNOLOGÍA E IMPLICACIONES DE LAS POLÍTICAS PÚBLICAS}

¿Cómo integrar la gestión de los sistemas de salud con políticas públicas que promuevan los ODS? El punto medular es lograr eficacia y retroalimentación en la integración multisectorial de dichas políticas.

El sistema de salud forma parte del sistema tecnológico, y la racionalidad que define su diseño y operatividad mejora con la capacidad técnica de sus operadores y de las poblaciones que cubre (18). Es fundamental reducir el gradiente tecnológico de manera eficaz, tanto al interior de los sistemas de salud como en su contexto social. Atender la transición epidemiológica requiere que la población mejore activamente su salud mediante la adopción de estilos de vida saludables (19). Lograr los ODS requiere que el sector de la salud promueva las capacidades tecnológicas de la población para proveer su bienestar y salud.

El financiamiento público de sistemas de salud permite alcanzar logros relacionados con la salud y facilita incidir sobre los DSS, de forma coordinada con las políticas públicas locales y nacionales. Esto puede incluir también la capacidad para lograr diagnósticos a niveles local y nacional adecuados. Así, el financiamiento público puede asignar recursos a las poblaciones a partir de su realidad tecnológica local, lo que contribuye a reducir las trampas de pobreza. Los criterios de equidad y costo-efectividad pueden aplicarse integralmente con la inclusión de objetivos específicos de difusión tecnológica para la reducción del gradiente tecnológico, posiblemente mediante la focalización de los subsidios públicos (20).

Un componente tecnológico importante para la operación contextualizada de los sistemas de salud son los sistemas de información fidedignos, relevantes y oportunos (21). Estos permiten monitorear las condiciones de bienestar y salud en las poblaciones e identificar brechas en la atención. Su utilización 
es clave para fines de planificación estratégica y de coyuntura, y constituye una extensión integrada de capacidades tecnológicas. La actual pandemia de la COVID-19 provee un ejemplo a través de la aplicación de pruebas de detección poblacionales que ilustra cómo una tecnología puede expandir capacidades de gestión.

\section{REFLEXIONES FINALES}

Lograr los objetivos de desarrollo y salud sostenibles es inseparable del desarrollo tecnológico y del incremento de las capacidades tecnológicas de la población más desfavorecida. Su rezago se debe a que en los sectores de pequeña escala, la innovación y la difusión tecnológicas tienen características de bien público y no son provistas eficientemente por el mercado, lo que hace que se rezague en un gradiente tecnológico. La experiencia histórica en políticas públicas de salud representa un acervo institucional singular en la creación de bienes públicos y en el mantenimiento de valores éticos igualitarios. Estos proveen un punto de partida y sostén para políticas públicas que reduzcan el gradiente tecnológico, con lo que se reduce la desigualdad mediante el uso de metodologías y criterios objetivos y eficientes, a fin de promover el bienestar y la salud de la población en general.

Contribución de los autores. Todos los autores concibieron el estudio original, escribieron el manuscrito, y revisaron y aprobaron la versión final.

\section{Conflictos de intereses. Ninguno a declarar.}

Declaración. Las opiniones expresadas en este artículo son responsabilidad de los autores y no reflejan necesariamente los criterios ni la política de la Revista Panamericana de Salud Pública / Pan American Journal of Public Health y/o de la Organización Panamericana de la Salud.

\section{REFERENCIAS}

1. United Nations General Assembly. Transforming our World: the 2030 Agenda for Sustainable Development (A/ RES/70/1). New York: UN; 2015 [citado el 19 de agosto de 2020]. Disponible en: https://www.un.org/en/development/desa/population/migration/generalassembly/docs/globalcompact/A_RES_70_1_E.pdf

2. Organización Panamericana de la Salud, Organización Mundial de la Salud. Agenda de Salud Sostenible para las Américas 2018-2030: un llamado a la acción para la salud y el bienestar en la Región de las Américas [Internet]. Washington, DC: OPS, OMS; 2017 [citado el 2 de agosto de 2020]. Disponible en: https:/ / iris.paho.org/bitstream/ handle /10665.2/49169/CSP296-spa.pdf?sequence=1\&isAllowed=y

3. Solar O, Irwin A. A conceptual framework for action on the social determinants of health. Geneva: World Health Organization; 2010. (Series on Social Determinants of Health Report No. 2).

4. Palmer RC, Ismond D, Rodríguez EJ, Kaufman JS. Social determinants of health: future directions for health disparities research. Am J Public Health. 2019;109(S1):S70-1.

5. Mayer-Foulkes D. Macroeconomics of poverty: Development and underdevelopment under globalization. Scotts Valley, CA: First Create Space Independent Publishing Platform; 2017.

6. Benhabib J, Perla J, Tonetti C. Reconciling models of diffusion and innovation: A theory of the productivity distribution and technology frontier. Cambridge, MA: National Bureau of Economic Research; 2017. (NBER Working Papers).

7. Hertog S. The association between two measures of inequality in human development: income and life expectancy. New York; United Nations; 2013 [citado el 2 de agosto de 2020]. (Technical Paper No. 2013/7). Disponible en: https://www.un.org/development/ $\mathrm{desa} / \mathrm{pd} /$ sites / www.un.org.development.desa.pd/files / files / documents/2020/Jan/un_2013_techpaper7.pdf

8. México, Instituto Nacional de Estadística, Geografía e Informática. Micro, pequeña, mediana y gran empresa. Estratificación de los establecimientos. Censos Económicos [Internet]. 2014 [citado el 6 de agosto de 2020]. Disponible en: http://internet.contenidos.inegi. org.mx/contenidos/productos / / prod_serv/contenidos/espanol/ bvinegi/productos/nueva_estruc/702825077952.pdf

9. United States Census Bureau. Number of Firms, Number of Establishments, Employment, and Annual Payroll by Enterprise Employment Size for the United States and States, Totals: 2017. 2020 [citado el 2 de agosto de 2020]. Disponible en: https:/ /www2. census.gov/programs-surveys/susb/tables/2017/us_naicssector_ large_rcptsize_2017.xlsx
10. Robert SA. Socioeconomic position and health: The independent contribution of community socioeconomic context. An Rev Sociol [Internet]. 1999;25(1):489-516 [citado el 2 de agosto de 2020]. Disponible en: http://www.annualreviews.org/doi/abs/10.1146/ annurev.soc.25.1.489?journalCode $=$ soc

11. Abramo L, Cecchini S, Ullmann H. Enfrentar las desigualdades en salud en América Latina: el rol de la protección social. Cien Saude Colet. 2020;25:1587-98.

12. Mújica ÓJ, Moreno CM. De la retórica a la acción: medir desigualdades en salud para no dejar a nadie atrás. Rev Panam Salud Publica. 2019;43:e12.

13. Marmot M, Friel S, Bell R, Houweling TAJ, Taylor S. Closing the gap in a generation: health equity through action on the social determinants of health. Lancet. 2008;372(9650):1661-9.

14. Graham H. Social determinants and their unequal distribution: Clarifying policy understandings. Milbank Q. 2004;82(1):101-24.

15. Case A, Lubotsky D, Paxson C. Economic status and health in childhood: The origins of the gradient. Am Econ Rev. 2002;92(5):1308-34.

16. Mayer-Foulkes D. The human development trap in Mexico. World Dev. 2008;36(5):775-96.

17. Mayer-Foulkes DA, Pescetto-Villouta C. Economic development and non-communicable chronic diseases. Glob Econ J. 2012;12(4):1-44.

18. Repullo Labrador JR, Segura Benedicto A. Salud pública y sostenibilidad de los sistemas públicos de salud. Rev Esp Salud Publica. 2006;80(5):475-82.

19. World Health Organization. From Alma-Ata to Astana: Primary health care-reflecting on the past, transforming for the future. Geneva: WHO; 2018.

20. Banco Mundial. Informe sobre el desarrollo mundial 1993: invertir en salud. Washington, DC: BM; 1993.

21. Plazzotta F, Luna D, González Bernaldo de Quirós F. Sistemas de información en salud: integrando datos clínicos en diferentes escenarios y usuarios. Rev Peru Med Exp Salud Publica. 2015;32(2):343-51.

Manuscrito recibido el 21 de mayo del 2020. Aceptado para publicación, tras revisión, el 17 de octubre del 2020. 


\section{The Sustainable Development Goals and Technological Capacity}

SUMMARY In order to achieve the Sustainable Development and Health Goals, it is essential to increase the technological capacity of the most disadvantaged populations. In the 21st century, the necessary technologies for this exist. The gap in technological capacity reflects the existence of a technological gradient between large- and small-scale production, due to an absence of incentives for innovation and a lack of technological dissemination in small businesses and communities. Technological change is central to development, but it is a public good that the market economy does not provide efficiently. Providing it requires the implementation of public policies aimed at technological innovation and dissemination. Reducing the technological gradient is therefore a major part of the United Nations 2030 Agenda for Sustainable Development and the Pan American Health Organization's 2018-2030 Sustainable Health Agenda for the Americas. This also applies to the development of health systems, which function as a redistribution mechanism to break poverty traps. In addition, experiences in these systems are relevant to the implementation of policies that increase technological capacities aimed at reducing poverty, improving social determinants of health, and thereby reducing the scale of the human development trap.

Keywords Science, technology and society; sustainable development; equity; health systems; health policy; social determinants of health.

\section{Os Objetivos de Desenvolvimento Sustentável e a capacidade tecnológica}

RESUMO Para alcançar os objetivos de desenvolvimento e saúde sustentáveis, é fundamental aumentar a capacidade tecnológica das populações mais desfavorecidas. Com a entrada do século XXI, há tecnologias necessárias para isso. O déficit em capacidade tecnológica decorre de um gradiente tecnológico entre a produção em pequena e larga escala pela falta de incentivos à inovação e difusão em empresas e comunidades pequenas. Nestas esferas, a evolução tecnológica, que é o eixo do desenvolvimento, é um bem público que não é provido de forma eficiente pela economia de mercado. Políticas públicas de inovação e difusão tecnológicas são necessárias. Diminuir o gradiente tecnológico constitui, portanto, a base da Agenda 2030 para o Desenvolvimento Sustentável, da Agenda das Nações Unidas e da Agenda de Saúde Sustentável para as Américas 2018-2030 da Organização Pan-Americana da Saúde. Requer também o desenvolvimento dos sistemas de saúde que servem como mecanismos de redistribuição para romper com as armadilhas da pobreza. Ademais, a experiência adquirida nesses sistemas é indispensável para instituir políticas de aumento da capacidade tecnológica que diminuam a pobreza, melhorem os determinantes sociais da saúde e, assim, reduzam a dimensão da armadilha do desenvolvimento humano.

Palavras-chave Ciência, tecnologia e sociedade; desenvolvimento sustentável; equidade; sistemas de saúde; política de saúde; determinantes sociais da saúde. 